\title{
Institutionen und Strukturen der Geschichtswissenschaft in Frankreich
}

\section{Pierre Monnet}

Traducteur : Niels F. May et Volker Zimmermann

\section{OpenEdition \\ Journals}

Édition électronique

URL : http://journals.openedition.org/ifha/7846

DOI : 10.4000/ifha.7846

ISSN : 2198-8943

Éditeur

IFRA - Institut franco-allemand (sciences historiques et sociales)

Référence électronique

Pierre Monnet, «Institutionen und Strukturen der Geschichtswissenschaft in Frankreich », Revue de

I'IFHA [Online], HS | 2014, Online erschienen am: 01 September 2014, aufgerufen am 03 Mai 2019.

URL : http://journals.openedition.org/ifha/7846 ; DOI : 10.4000/ifha.7846

Ce document a été généré automatiquement le 3 mai 2019.

(CIFHA 


\title{
Institutionen und Strukturen der Geschichtswissenschaft in Frankreich
}

\author{
Pierre Monnet
}

Traduction : Niels F. May et Volker Zimmermann

\section{NOTE DE L'ÉDITEUR}

Übersetzt von Niels F. May und Volker Zimmermann

\section{Einleitung}

1 Kurz vor dem Fall der Berliner Mauer 1989 veröffentlichten Jacques Leenhardt und Robert Picht 100 Schlüsselbegriffe für Deutsche und Franzosen (französischer Titel : Au jardin des malentendus) zum intellektuellen Austausch zwischen Frankreich und Deutschland ${ }^{1}$. Unter den vielen Beiträgen zu den Gemeinsamkeiten und Unterschieden zwischen der deutschen und französischen Kultur befand sich auch ein Artikel zur Wissenschaftspolitik ${ }^{2}$. Schon $\mathrm{zu}$ diesem Zeitpunkt stellte sich dabei anhand der Statistiken heraus, dass die Ausgaben für Forschung und Technologie in Deutschland höher waren - und zwar sowohl in absoluten Zahlen als auch in Bezug auf den Anteil am Bruttoinlandsprodukt (in Frankreich 2,25\% des BIP und in 2,80\% in Westdeutschland). Außerdem zeigte die Studie auf, dass diese Ausgaben in Frankreich zu gleichen Teilen auf die öffentliche und private Hand (vor allem Unternehmen) verteilt waren, während in Deutschland lediglich $38 \%$ der Ausgaben auf die öffentliche Hand entfielen und $62 \%$ auf Unternehmen. In der Gesamtschau wurde darüber hinaus die Rolle betont, die private Stiftungen bei der Forschungsförderung und -finanzierung in Deutschland hatten und haben. Herausgestellt wurde schließlich auch die zentrale Rolle von Staat und Zentralregierung in der französischen Forschungspolitik, während in Deutschland die 
entsprechenden Kompetenzen und Anregungspotentiale - über das Instrument von zeitlich begrenzten und durch Begutachtung evaluierten Projekten - zwischen Ministerien, den Ländern, außeruniversitären Einrichtungen und den Universitäten selbst aufgeteilt waren.

Wo stehen wir ein Vierteljahrhundert später, nach der Wiedervereinigung Deutschlands 1990, der Einführung des Euro (1999-2002), dem Beginn des Bologna-Prozesses im Jahr 1999, auf den 2010 die Einrichtung des Europäischen Hochschulraums folgte, nach der im Jahr 2000 verabschiedeten Lissabon-Strategie (mit dem Ziel, Europa zum wettbewerbsfähigsten Wissensmarkt der Welt zu machen), nach den tiefgreifenden Reformen der europäischen Universitäts- und Forschungsstrukturen im letzten Jahrzehnt, nach der Entwicklung von europäischen Programmen wie dem Forschungsrahmenprogramm (FRP), das 1983 gestartet wurde, und nach der Schuldenund Finanzkrise der öffentlichen Haushalte?

Die hier gegebenen Antworten auf diese Frage konzentrieren sich auf die französische Seite und beschränken sich darüber hinaus auf die Strukturen der Geschichtswissenschaft, wie es dem Ziel dieses Wegweisers entspricht. Für die deutsche Seite sei auf den im französischen Pendant erschienenen Beitrag von Falk Bretschneider, Bernd Klesmann und Rahul Markovits zu den Strukturen der Geschichtswissenschaft in Deutschland verwiesen, der den bezeichnenden Untertitel »Universités, instituts de recherche extra-universitaires et associations « trägt ${ }^{3}$. Es mag dennoch hilfreich sein, hier noch einmal in groben Zügen die deutsche Forschungslandschaft zusammenzufassen, um vor diesem Hintergrund besser die Unterschiede zum französischen System herausarbeiten zu können. Die Autoren unterstreichen vor allem die Unabhängigkeit der Universitäten im föderalen System, das den Ländern die alleinige Verantwortung für diese Institutionen zuteilt, wenn auch im Zeichen der Exzellenzinitiative seit einigen Jahren der forschungspolitische Einfluss des Bundes wieder zunimmt. Sie führen außerdem an, dass sich die deutschen Universitäten nach wie vor am Ideal einer auf dem neuesten Forschungsstand basierenden Lehre orientieren. Eine zentrale Rolle spielen hierbei die Lehrstühle, deren Inhaber allein verbeamtet sind - ein Status, der erst nach einer langen Durststrecke aus prekären Anstellungsverhältnissen und befristeten Projektverträgen erreicht werden kann. Sie erwähnen auch die vielfältigen Finanzierungsmöglichkeiten, die der historischen Forschung in Deutschland jenseits von Bundes- und Länderhaushalten zur Verfügung stehen: Dazu zählen die Deutsche Forschungsgemeinschaft (DFG), die großen privaten Stiftungen, außeruniversitäre Institute wie die Max-Planck-Gesellschaft, Forschungsbibliotheken (zum Beispiel die Bayerische Staatsbibliothek in München oder die Herzog-August-Bibliothek in Wolfenbüttel), die historischen Museen (deren Gewicht sich an der Publikumswirksamkeit bemisst, die große historische Ausstellungen in Deutschland haben können) und die großen historischen Institute im Ausland (wie das Deutsche Historische Institut in Paris). 


\section{Die Rolle der Geistes- und Sozialwissenschaften in der französischen Forschung unter besonderer Berücksichtigung der Geschichtswissenschaft}

Es ist vielleicht angebracht, mit einigen Zahlen und Strukturelementen der französischen Hochschul- und Forschungslandschaft zu beginnen ${ }^{4}$. Frankreich hat $201128 \mathrm{Mrd}$ € für die Hochschulbildung aufgewendet, wobei zwei Drittel des Betrags auf Gehaltszahlungen entfallen und davon wiederum ein Großteil auf die Gehälter der Hochschullehrer (insgesamt 56.500, davon 4.500 Professoren und 10.800 maitres de conférences in den Geistes- und Sozialwissenschaften). 72 \% der Ausgaben für die Lehre an den französischen Universitäten werden vom Staat finanziert. Die Forschungsausgaben, berechnet nach internationalen Standards unter Berücksichtigung der Inlandsausgaben für Forschung und Entwicklung, erreichen 45 Mrd. $€$, was $2,25 \%$ des BIP entspricht. Im Vergleich dazu widmet Deutschland diesem Sektor 2,89 \% des BIP, was den $3 \%$ sehr nahe kommt, die von der im Jahr 2000 verabschiedeten Lissabon-Strategie (zum Aufbau eines wissensgestützten Wirtschaftsraumes in Europa) für alle Mitgliedsstaaten der Europäischen Union empfohlen werden.

5 In Frankreich werden die Ausgaben für Forschung und Entwicklung hauptsächlich von Unternehmen getragen, deren Anteil 63 \% beträgt. Der Anteil der öffentlichen Hand liegt bei 15,9 Mrd. $€$ und wird vor allem durch die großen öffentlichen Forschungs- und Bildungseinrichtungen aufgebracht - $53 \%$ dieses Betrags entfallen auf öffentliche Forschungsorganisationen, $29 \%$ auf Universitäten und öffentliche Bildungseinrichtungen und $18 \%$ auf das Centre National de Recherche Scientifique (CNRS), dessen Budget 3,077 Mrd. € beträgt. Selbstverständlich ist im zweiten (d.h. öffentlichen) Bereich der Anteil der Geistes- und Sozialwissenschaften - und damit auch der Geschichtswissenschaft - am größten, da diese Disziplin kaum von Patentanmeldungen, gewinnorientierten Public-Private-Partnerships, Technologietransfer, Militärausgaben, Investitionen der regionalen Gebietskörperschaften oder Steuervergünstigungen für die Forschung betroffen ist.

6 Geschichtswissenschaftliche Forschung findet also wesentlich im öffentlichen Sektor statt, das heißt an Universitäten, in nationalen Hochschulinstitutionen wie der École des Hautes Études en Sciences Sociales (EHESS), der École Pratique des Hautes Études (EPHE), der École Nationale des Chartes (ENC) oder den Écoles Normales Supérieures (ENS) sowie in großen öffentlichen Forschungsorganisationen wie dem CNRS, die allesamt durch die vor einigen Jahren eingerichteten 25 Cluster oder PRES (Pôles de Recherche et d'Enseignement Supérieur) stärker miteinander verknüpft werden sollen. Die PRES wiederum sind aktuell Veränderungen unterworfen und sollen bis Ende 2014 in stärker institutionalisierte Gemeinschaften oder COMUEs (Communautés d'universités et d'établissements) überführt werden ${ }^{5}$.

7 Wie es auch in anderen Disziplinen der Fall ist, sind Historiker vor allem in Paris und Umgebung angesiedelt. In dieser Region befinden sich neben einer erheblichen Anzahl von Universitäten neun der PRES bzw. zukünftigen COMUEs, große öffentliche Institutionen wie die EHESS, die EPHE, die ENC und zwei ENS (rue d'Ulm in Paris und Cachan), die Zentralverwaltung des CNRS, zahlreiche Archive und große Bibliotheken (wie z.B. die französische Nationalbibliothek BnF). Dies erklärt, warum von den 96.000 
Vollzeitstellen für Forscher im öffentlichen Dienst (davon $50 \%$ an Universitäten und $50 \%$ in anderen Institutionen und öffentlichen Einrichtungen) 34.000 in der Region Île-deFrance angesiedelt sind, gefolgt von Lyon mit 12.000 Vollzeitstellen im öffentlichen Dienst.

8 Von den 56.500 Hochschullehrern entfallen $31 \%$ auf die Geistes- und Sozialwissenschaften. Hinzu kommt eine große Endogamie in der Rekrutierung : 40 \% der maitres de conférences haben vor ihrer Einstellung zwar in der Forschung gearbeitet (als Doktoranden, Postdoktoranden oder chargés de recherche), nur $5 \%$ der Universitätsprofessoren aber werden direkt aus der Forschung übernommen, d.h., ohne dass sie eine Zwischenstation in einer Universität durchlaufen haben.

Zum Abschluss dieses statistischen Überblicks einige Zahlen zu den Doktoranden : 2012 waren 21.805 Doktoranden in den Geisteswissenschaften eingeschrieben ( "Lettres et Sciences Humaines« nach der Nomenklatur des französischen Hochschulministeriums), was $35 \%$ der Doktoranden aller Disziplinen entspricht. Von den rund $12.000 \mathrm{im}$ Jahr 2012 abgeschlossenen Promotionen entfallen rund ein Viertel auf die Geistes- und Sozialwissenschaften. Alle Doktoranden müssen in einer Doktorandenschule (école doctorale) eingeschrieben sein, deren Zahl über alle Disziplinen hinweg bei der letzten Erfassung 2012286 betrug. Bei den von französischen Forschern geleiteten Projekten im Rahmen des 7. Forschungsrahmenprogramms (FRP) der Europäischen Union sind Geisteswissenschaftler unterrepräsentiert ${ }^{6}$, und auch die internationale Sichtbarkeit von französischsprachigen wissenschaftlichen Veröffentlichungen in den Geistes- und Sozialwissenschaften ist nach wie vor beschränkt.

Die historische Forschung stellt also einen nicht zu vernachlässigenden Bereich innerhalb des größeren Ensembles der Geisteswissenschaften dar. Die wissenschaftlichen Aktivitäten in diesem Bereich hängen zum Großteil vom öffentlichen Sektor ab und werden mit öffentlichen Mitteln finanziert. Getragen werden sie zum einen von Beschäftigten mit Zeitverträgen, zum anderen aber - und zum ganz überwiegenden Teil von verbeamteten Forschern, die als Hochschullehrer (enseignants-chercheurs) an den Universitäten und anderen Hochschulen tätig sind bzw. als wissenschaftliche Mitarbeiter und Forschungsdirektoren (chargés de recherche und directeurs de recherche) an außeruniversitären Forschungseinrichtungen.

Der Einstieg in den Forschungs- und Universitätssektor findet, wie auch in anderen Ländern, über die Promotion und die Post-doc-Phase statt, d.h. auf der Basis eines befristeten Arbeitsverhältnisses oder ohne jegliche vertragliche Anbindung. Zwar ist die Finanzierung während der Promotion in Frankreich durch Graduiertenkollegs und Promotionsstipendien teilweise gesichert (diese Finanzierung deckt jedoch nur drei Jahre $\mathrm{ab}$ und kommt durchschnittlich $66 \%$ der eingeschriebenen Promotionsstudierenden zugute), die Post-doc-Phase hingegen bleibt bei weitem die prekärste Etappe auf dem Weg zur einer Forscherstelle, was durch die immer seltener werdenden Festanstellungen in Universitäten oder Forschungseinrichtungen noch verschärft wird. Im Gegensatz zu Deutschland steht jedoch jeder Doktorand oder Postdoktorand, der eine Finanzierung, ein Stipendium oder eine anderweitige institutionelle Unterstützung erhält, in einem Arbeitnehmerverhältnis. Frankreich folgt hier der EU-Empfehlung, ein Promotionsstipendium als einen sozial- und rentenversicherungspflichtigen Arbeitsvertrag anzusehen. 


\section{Die Forschung inner- und (zunehmend auch) außerhalb der Universität}

12 In Frankreich gibt es heute 86 Universitäten, die alle mehr oder weniger intensiv Forschung betreiben. Im Gegensatz $\mathrm{zu}$ Deutschland liegen die französischen Universitäten nicht im Verantwortungsbereich der Regionen, sondern unterstehen hinsichtlich ihrer Finanzierung und der Dienstaufsicht allein dem nationalen Ministerium für Bildung, Hochschulbildung und Forschung (Ministère de l'Éducation nationale, de l'Enseignement Supérieur et de la Recherche) ${ }^{7}$. Wie der Name des Ministeriums erkennen lässt, ist es Regierungspolitik, dass Universitäten und Forschung zumindest theoretisch von den gleichen Instanzen geleitet werden und den gleichen Zielsetzungen unterliegen.

13 Fast alle französischen Universitäten verfügen mindestens auf dem licence-Niveau (entspricht dem deutschen bzw. europäischen Bachelor) über einen Studiengang in Geschichte, der von einem fest angestellten Professor betreut wird, welcher in der Lage ist, in seiner Einrichtung oder anderswo Forschungsarbeiten zu betreuen und bestimmte Studierende auf die nationalen Auswahlverfahren (concours) vorzubereiten ${ }^{8}$. Rechtlich gesehen sind die Universitäten öffentliche Einrichtungen mit wissenschaftlichem, kulturellem und berufsbildendem Charakter (Établissement Public à Caractère Scientifique, Culturel et Professionnel oder EPCSCP). In diese Kategorie fallen auch die grands établissements genannten großen, nationalen Hochschuleinrichtungen wie die EHESS $^{9}$ und die EPHE $^{10}$, die auf Forschung und Lehre ab dem Master-Niveau spezialisiert sind, sowie die französischen Forschungseinrichtungen im Ausland (Écoles françaises à l'étranger, EFE) und die Écoles Normales Supérieures (ENS), die in Zusammenarbeit mit einer Universität Master- oder Doktorabschlüsse vergeben können.

Der Zugang zu diesen grandes écoles ist nur über Auswahlverfahren möglich, und ihre Studierenden werden für die Zeit des Studiums verbeamtet. Die EPCSCPs sind von den öffentlichen Einrichtungen mit wissenschaftlichem und technologischem Charakter (Établissement Public à caractère Scientifique et Technologique, EPST) zu unterscheiden. Für die Geschichtswissenschaft und die ihr verwandten Disziplinen sind in diesem Bereich beispielswiese relevant das in seiner heutigen Form 1939 gegründete $\mathrm{CNRS}^{11}$ oder das Institut National d'Études Démographiques (INED) ${ }^{12}$.

Beschränkt man sich auf die jüngere Vergangenheit, dann wurde Geschichtswissenschaft in Frankreich zuerst an jenen Universitäten betrieben, an denen ab 1808 historische Lehrstühle in den von Napoleon im Rahmen der Université impériale neu eingerichteten geisteswissenschaftlichen Fakultäten entstanden ${ }^{13}$. Die herausgehobene Stellung dieser Lehrstühle erklärt sich auch durch die agrégation d'histoire (Staatsexamen in Geschichte für den Sekundarschulbereich), die 1830 eingerichtet wurde und seitdem den Königsweg einer Universitätskarriere darstellt. Diese historisch gewachsene Verbindung zwischen agrégation und Hochschullehre ist auch heute noch wirkmächtig und zeigt sich etwa an dem Platz, den Hochschullehrer in der Prüfungskommission der agrégation einnehmen, oder der Zahl von agrégés, die an den Universitäten als ATER (attaché temporaire d'enseignement et de recherche - zeitlich befristete Lehr- und Forschungsstellen, wovon $34 \%$ in geistes- und sozialwissenschaftlichen Fakultäten unterrichten), PRAG (professeurs agrégés = Lehrer im Hochschuldienst), Promotionsstipendiaten oder Lehrbeauftragte (in Voll- oder Teilzeit oder in der Form von Stundenverträgen) beschäftigt sind. 
16 Anders als in Deutschland, wo Forschung und Lehre über den Lehrstuhl stark miteinander verbunden sind, war dieses Verhältnis in Frankreich in der Vergangenheit aus mehreren Gründen nicht besonders eng: Dazu zählen die Konzentration auf die Lehre und die Auswahlverfahren (concours), aber auch die frühe Politisierung der Historiker im 19. Jahrhundert (sei es auf der Seite der Monarchisten oder auf der Seite der Erben der Französischen Revolution von 1789), ihr nationalgeschichtliches Engagement, das sie fleißig an den großen Meistererzählungen der Nation stricken und ihren Teil zur Patrimonialisierung der Nationalgeschichte beitragen ließ, sowie ihre Rolle im Konkurrenzkampf mit dem Deutschen Kaiserreich, das 1870 aus dem Sieg über Frankreich hervorgegangen war und von einer vermeintlichen ideologischen und intellektuellen Überlegenheit überstrahlt wurde, die es gleichermaßen zu imitieren und zu begrenzen galt.

Alle diese Elemente erklären, warum die gesellschaftliche Funktion der Geschichte innerhalb der französischen Universität des 19. Jahrhunderts, aber auch die Professionalisierung der Geschichtswissenschaft, die Bedeutung der Lehre und der Beziehung zwischen Lehrer und Schülern, die Obsession der agrégation oder die untrennbare Verbindung zwischen Geschichte und Geographie - für alle diese Aspekte lässt sich auf die Übervater-Figur von Ernest Lavisse (1842-1922) verweisen - nicht zu einer stärkeren Verknüpfung von Forschung und Lehre geführt haben. Vereinfacht gesagt, fanden am Ende des 19. Jahrhunderts kritische Forschung und eine Erneuerung der traditionellen Gelehrsamkeit in Geschichte ihren Platz an der 1794 gegründeten École Normale Supérieure ${ }^{14}$, der 1821 gegründeten École des Chartes ${ }^{15}$ und der 1868 gegründeten École Pratique des Hautes Études ${ }^{16}$.

Diesen Institutionen gegenüber standen im Jahr 191455 Lehrstühle, die die französische historische Schule verkörperten, für die Geschichte die Schule des Citoyen ist, ein Instrument zur politischen Bildung, das sich in einer pädagogisch orientierten Nationalgeschichtsschreibung niederschlägt. Der Erste Weltkrieg bot bekanntlich ebenfalls die Gelegenheit einer beschleunigten Infragestellung der Disziplin und ihrer akademischen Vertreter durch die Soziologie und die Anthropologie sowie - im Gewand der Annales-Schule - durch eine als Sozialwissenschaft verstandene Geschichte. Diese Infragestellung betraf sowohl den Beruf des Historikers als auch die Methoden und die Ziele der Historiographie, wandte sich aber stärker gegen die traditionelle Universität als gegen die Geschichtswissenschaft selbst ${ }^{17}$.

Das CNRS, das 1939 aus der Fusion der 1935 gegründeten Caisse Nationale de la Recherche Scientifique und des 1938 gegründeten Centre National de la Recherche Scientifique Appliquée hervorging, bildete daraufhin den Ort, an dem sich neue Forschungstendenzen oder Disziplinen ansiedelten, die an der Universität nur schwer einen Platz fanden (etwa Archäologie, Kunstgeschichte, Disziplinen der außereuropäischen Welt oder die kritischfundierte Erforschung von Schrift und Schriftlichkeit am 1937 gegründeten Institut de Recherche et d'Histoire des Textes IRHT) ${ }^{18}$.

\section{Erste Neuordnungen nach 1945}

Damit die historischen Lehrstühle an den Universitäten - nicht ohne Widerstand - von den Diskussionen um die Mentalitätsgeschichte, den Strukturalismus, die »nouvelle histoire« oder die historische Anthropologie profitierten, brauchte es eine völlige 
Flurbereinigung der gesamten Forschungs- und Hochschullandschaft Frankreichs : Nach 1945 stieg der Einfluss des CNRS, im gleichen Jahr wurde das INED gegründet, ab 1947 schuf Fernand Braudel von der 6. Sektion (Wirtschafts- und Sozialwissenschaften) der EPHE ausgehend nach und nach ein veritables Imperium im Bereich der Sozialwissenschaften und der Geschichte (erster Präsident dieser Sektion war 1948 Lucien Febvre, Braudel ihr wissenschaftlicher Sekretär, bevor er 1949 das Centre de Recherches Historiques gründete und die 6. Sektion sich 1975 als École des hautes études en sciences sociales abspaltete).

21 In diesem Aufschwung der Geistes- und Sozialwissenschaften (in dessen Genuss auch die Historiker kommen, deren Zahl - Universitäten eingeschlossen - zwischen 1945 und 1967 von 3.000 auf 8.000 steigt) haben es die Universitäten angesichts der allgemeinen Explosion der Studierendenzahlen (1960 sind 309.000 Studierende in Frankreich eingeschrieben, 1970 850.000, 1980 1,2 Millionen, 1990 1,7 Millionen, 2000 2,1 Millionen und 2012 2,4 Millionen) dennoch schwer, auf dem Gebiet der Forschung mit den großen nationalen Forschungseinrichtungen und -organisationen wie dem CNRS mitzuhalten, das z.B. 1978 das zeithistorische Institut d'Histoire du Temps Présent (IHTP ${ }^{19}$ gründet. Gleichzeitig vermehren sich die Forschungszentren des Institut d'Études Politiques (gegründet 1945) in den 1970er und 80er Jahren stark.

An den Universitäten selbst fand die Forschung nach wie vor an den Lehrstühlen statt, die von Professoren und ihren Assistenten bzw. später den maitres de conférences (dieser Stellentyp wurde 1984 geschaffen) geleitet wurden. Die Anzahl der Professoren und Assistenten in Geschichte verzehnfachte sich an den französischen Universitäten zwischen 1950 und 1985 von 200 auf 2.000. Dieser Anstieg hielt auch in den 1990er Jahren an und flankierte im Rahmen des sogenannten Entwicklungsplans "Universität 2000 , der 1990 verabschiedet wurde, die steigenden Studentenzahlen. Die unternommenen finanziellen Anstrengungen (acht neue Universitäten wurden geschaffen, davon vier in der Pariser Region) folgten jedoch eher einer Logik der Quantität und stärkten die Forschung kaum.

Die steigende Zahl von Hochschullehrerstellen ging mit der Vermehrung von provisorischen und prekären Stellen einher, sodass die Lehrstühle nicht mehr in der Lage waren, die Wissenschaftslaufbahnen des Nachwuchses mit klaren forschungsstrategischen Orientierungen zu verbinden. Vorherrschend waren vielmehr eher eine gewisse Zersplitterung und die Entstehung kleinerer Gruppen und Forschungsinstitute um Zeitschriften, Datenbanken und Projekte herum, die den geographischen Rahmen der Universitäten sprengten. Dieses Phänomen dauert zum Teil bis heute an.

24 Es wäre jedoch falsch zu behaupten, die Universitäten hätten über diese Entwicklungen hinweg ihre wissenschaftlichen Profile nicht aufrechterhalten können. Neben den zwei Schwergewichten Paris und Lyon, wo so gut wie alle Bereiche der historischen Forschung vertreten sind, richten die geschichtswissenschaftlichen Fachbereiche von Nancy und Straßburg (wo in der Mediävistik einst Marc Bloch lehrte, aber auch Charles-Edmond Perrin sowie Robert Folz, der seine Karriere in Dijon beendete) ihre Aufmerksamkeit besonders auf die deutschsprachige Welt, während die Fachbereiche in Toulouse und Bordeaux traditionellerweise eher auf die spanische Geschichte spezialisiert sind, die historischen Institute in Nizza, Grenoble und Montpellier vor allem nach Italien schauen und der Fachbereich in Caen sich bevorzugt mit dem anglophonen Raum beschäftigt. Darüber hinaus verfügen die Germanistik-Fachbereiche einiger Universitäten (Paris- 
Sorbonne IV, aber auch Paris Sorbonne Nouvelle III, Lille, Metz, Nancy, Straßburg und Lyon) im Bereich der civilisation germanique über Forschungsprogramme zur Geschichte Deutschlands ${ }^{20}$.

Keine dieser Universitäten aber hat es in diesem Zeitraum vermocht, tatsächlich die parallel existierenden Forschungseinrichtungen zu ersetzen. Die Folge ist, dass die Definition der Aufgaben und des Berufs des Historikers zwischen der akademischen Welt der Universitäten und der von der Lehre weitgehend abgekoppelten Welt der Forschung weiterhin unterschiedlich ausfällt und immer wieder zum Zankapfel wird ${ }^{21}$. Ein Zeichen für diese »feinen Unterschiede« ist beispielsweise der Umstand, dass die Lehrstühle an der EHESS oder der EPHE, aber auch am Collège de France, nach dem Forschungsgebiet ihres Inhabers benannt werden (im Laufe einer Karriere können sie also auch umbenannt bzw. nach dem Weggang des Stelleninhabers mit einem neuen Titel versehen werden), während die Lehrstühle an den Universitäten weiterhin mehrheitlich nur die Epochenbezeichnungen tragen - selbst wenn es Ausnahmefälle gibt, bei denen einzelne Lehrstühle thematisch ausgerichtet sind. Der Normalfall ist dies aber nicht.

Im Unterschied zu Deutschland fällt ebenfalls auf, dass nur sehr wenige Lehrstühle explizit einer diachron vorgehenden Regionalgeschichte gewidmet sind (Ausnahmen bestehen in einigen "regionalistischen« Bereichen wie der okzitanischen, bretonischen, korsischen, baskischen und elsässischen Geschichte, die allesamt aber Randphänomene darstellen). Mit Blick auf die inzwischen »alten« Beispiele wie Lucien Febvre, Emmanuel Leroy-Ladurie, Pierre Chaunu oder Georges Duby muss jedoch unterstrichen werden, dass regionalgeschichtliche Arbeiten - vor allem im Rahmen monographischer Forschung immer von Lehrstühlen bzw. Fachbereichen mit allgemeingeschichtlicher Orientierung ausgegangen sind, also nicht von spezifisch auf die »Region« ausgerichteten Einheiten. Die französische Geschichte unterscheidet sich auch hier deutlich vom deutschen Fall und seiner landesgeschichtlichen Tradition.

Umgekehrt gibt es in Frankreich keine allgemein ausgerichteten, periodenübergreifend arbeitenden Lehrstühle, die sich mit Universal- oder Globalgeschichte beschäftigen. Auch wenn sich die Umstände in Frankreich seit den 1990er Jahren langsam zu ändern begonnen haben, gab es hier keine periodenübergreifende und interdisziplinäre Strukturierung der Geschichtswissenschaft, wie sie in Deutschland zwischen Fachbereichen und Fakultäten einer gleichen Universität durch die Sonderforschungsbereiche hat geschehen können ${ }^{22}$. Dies erklärt zum Teil auch die immer noch anhaltenden hartnäckigen Blockaden zwischen der Geschichtswissenschaft und anderen benachbarten Disziplinen wie der Archäologie, der Kunstgeschichte und der Literaturwissenschaft.

Die disziplinäre Nachbarschaft mit der Theologie ist in Frankreich ein Spezialfall, da dieses Fach seit 1885 nur noch außerhalb der staatlichen Universitäten gelehrt wird (ein Umstand, der durch das Gesetz über die Trennung von Staat und Kirche von 1905 noch einmal verstärkt wurde), und zwar in "Instituts catholiques « genannten, separaten Universitäten, die alle nach 1875 gegründet wurden, etwa in Lille ${ }^{23}$, Paris ${ }^{24}$ und Lyon ${ }^{25}$. Umgangssprachlich »La catho " genannt, beherbergen diese Einrichtungen aber auch andere, als »freie« bezeichnete Fakultäten neben der Theologie, z.B. in Jura, Medizin, Verwaltungs- oder Geisteswissenschaften. Außerhalb von Bachelor- und Masterstudiengängen bieten diese Institute innerhalb ihrer Forschungszentren auch Promotionen und Post-doc-Programme an, darunter mehrere in Geschichte des kanonischen Rechts, Exegese oder Patristik. 
29 Ein anderer Teil der theologischen Lehre und Forschung der alten Pariser Universität ist unter der Bezeichnung »Religionswissenschaften« zur 5. Sektion der École Pratique des Hautes Études geworden. Einzig das Elsass und das Departement Moselle bilden eine Ausnahme von der Regel der Abtrennung theologischer Lehre und Forschung: Beide gehörten von 1871 bis 1918 zum Deutschen Kaiserreich und unterliegen seit 1919 dem Konkordatsrecht, das im restlichen Frankreich nur bis 1870 galt. Die beiden Fakultäten für katholische und protestantische Theologie in Straßburg sind deshalb bis heute Teil der geisteswissenschaftlichen Universität Straßburg. Im Bereich der protestantischen Theologie sind darüber hinaus noch die freien, von den Universitäten unabhängigen Fakultäten von Paris und Montpellier zu erwähnen.

\section{Die Restrukturierung der französischen Universitäts- und Wissenschaftslandschaft seit den 1990er Jahren}

30 Trotz zahlreicher Kontinuitätslinien haben in der jüngsten Vergangenheit drei Entwicklungen zu einer entscheidenden Veränderung der Situation geführt :

31 1. In den 1990er Jahren reformierte das CNRS zusammen mit dem Ministerium für Forschung die Organisationsform der Unité Mixte de Recherche (UMR) ${ }^{26}$, die je eine Forschungseinheit des CNRS mit einer Universität verbindet, und weitete sie aus, was zu einer Vervielfältigung solcher Forschungsstrukturen führte. Auch in der Geschichtswissenschaft - bekannte UMRs hier sind etwa das Centre de Recherches Historiques $(\mathrm{CRH})^{27}$ an der EHESS oder das Laboratoire de Médiévistique Occidentale de Paris (LAMOP) an der Universität Paris 1 Panthéon-Sorbonne ${ }^{28}$ - haben diese Kooperationsformen neue Perspektiven erschlossen und zur Erneuerung der Forschung in einer ganzen Reihe von Feldern geführt, etwa der Geschichte der Politik und der internationalen Beziehungen ${ }^{29}$, der Kulturgeschichte ${ }^{30}$, der Geschichte der Archäologie ${ }^{31}$, der Umweltgeschichte ${ }^{32}$, der Wissenschaftsgeschichte ${ }^{33}$, der Kunstgeschichte ${ }^{34}$ und der Geschichte der Emotionen ${ }^{35}$. Neben diesen thematischen Erweiterungen standen auch regionale Forschungsschwerpunkte, etwa $\mathrm{zu}$ Spanien $^{36}$, Deutschland ${ }^{37}$, dem Mittelmeerraum ${ }^{38}$ oder Südostasien ${ }^{39}$.

In den gleichen Kontext einer Annäherung von CNRS und Universitäten gehört auch die 2007 erfolgte Gründung der thematischen Forschungscluster RTRA (Réseaux Thématiques de Recherche Avancée $)^{40}$, deren Ziel es ist, benachbarten und z.B. über einen PRES verbundenen Universitäten, grandes écoles und Forschungseinrichtungen zu erlauben, ihre Forschungsaktivitäten thematisch zu bündeln und zu koordinieren. In den Geistesund Sozialwissenschaften dominieren dabei zurzeit vor allem die Wirtschaftswissenschaften (die Wirtschaftsgeschichte eingeschlossen), die mit der École d'Économie de Toulouse ${ }^{41}$ und der École d'Économie de Paris ${ }^{42}$. die Vorreiterrolle einnehmen.

Die RTRAs haben ebenso zur Gründung eines Netzes von vier Instituts d'Études Avancées (Réseau français des Instituts d'études avancées, RFIEA) ${ }^{43}$ geführt: Dazu gehören das Collegium in Lyon, das sich mit der Diffusion von Wissen auseinandersetzt ${ }^{44}$, das Institut Méditérranéen des Recherches Avancées (IMéRA) in Marseille, das sich mit den menschlichen Grundlagen von Wissenschaft beschäftigt ${ }^{45}$, ein Institut in Nantes zur Globalgeschichte der Menschheit ${ }^{46}$ und ein Institut in Paris, das Fragen der Universalität 
erforscht ${ }^{47}$. Aufgabe dieser Institute ist sowohl die Nachwuchsförderung durch die Einrichtung von jungen Forschergruppen als auch die Organisation von Gastaufenthalten von ausländischen Wissenschaftlern.

Die Annäherung zwischen CNRS und Universitäten ist also eine der entscheidenden Entwicklungen, aber auch eine der großen aktuellen Herausforderungen, sowohl in Bezug auf die Arbeitsbedingungen als auch die strategische Forschungspolitik in Frankreich. Einer der Hauptträger dieser Entwicklung ist das Institut National des Sciences Humaines et Sociales (INSHS) mit 1.699 Forschern des $\mathrm{CNRS}^{48}$, 6.747 Hochschullehrern, 11.886 Doktoranden und 866 Postdoktoranden in den Geistes- und Sozialwissenschaften, die sich auf 258 UMRs und rund 100 Forschungsinstitute im Ausland verteilen, und einem Jahresbudget von 19 Millionen Euro im Jahr $2013^{49}$. An diesem Beispiel sieht man sehr deutlich, dass das CNRS immer noch ein zentraler Akteur in der geistes- und sozialwissenschaftlichen Forschung und insbesondere in der Geschichtswissenschaft ist. Diese Rolle zeigt sich auch daran, dass das CNRS an einer Politik der Delegation festhält, die es Hochschullehrern an Universitäten erlaubt, Forschungsfreisemester am CNRS zu nehmen und für diese Zeit von ihren Lehrverpflichtungen freigestellt zu werden. Deutlich wird sie aber auch durch die Einbindung des CNRS in das 2006 gegründete Groupement d'Intérêt Scientifique (GIS), welches die 22 Maisons des Sciences de l'Homme (MSH) ${ }^{50}$ zusammenfasst, die nach dem Modell der ersten Maison des Sciences de l'Homme -1962 von Fernand Braudel in Paris in Verbindung mit einer entsprechenden Stiftung (Fondation Maison des Sciences de l'Homme, FMSH) ${ }^{51}$ gegründet - in den letzten Jahrzehnten überall in Frankreich entstanden sind. Ursprüngliches Ziel dieser Einrichtungen war es, dort $\mathrm{zu}$ intervenieren, wo Universitäten und Forschungsorganisationen allein nur schwer agieren konnten (d.h. vor allem auf der Ebene der internationalen Forschungskooperation) und transversale, interdisziplinäre Forschungsthemen sowie innovative Forschungsinstrumente zu fördern.

2. Die zweite wichtige Entwicklung der letzten 15 Jahre besteht in der französischen Version einer sogenannten Exzellenzinitiative. Politisch ging diese auf eine ganze Reihe von Modernisierungsmaßnahmen zurück, die unter dem Label »Université 2000« (1990), »Université du $3^{e}$ millénaire $(2000-2006)$ auf der Basis von Verträgen zwischen Staat und Regionen sowie »Plan Campus« (2008) mit der Einrichtung der PRES firmierten, bevor sie 2010 in die durch eine große Staatsanleihe finanzierte Exzellenzinitiative mündeten ${ }^{52}$. Pate stand dabei - wie auch bei der Einrichtung der Evaluationsagentur AERES (Agence d'Évaluation de l'Enseignement Supérieur) und der nationalen Forschungsagentur ANR (Agence Nationale de la Recherche) - die deutsche Exzellenzinitiative. Ihr zur Seite stand zudem eine »investissements d'avenir« genannte Investitionsinitiative, die einer grundlegenden Erneuerung der französischen Universitätscampusse gewidmet war.

Drei Förderlinien zeichneten die französische Exzellenzinitiative aus : »Equipex« (für » équipements d'excellence«) zur Ausstattung von Forschungslaboratorien mit Großgeräten und Spitzentechnologie, »Labex (für »laboratoires d'excellence«) zur Förderung von Forschungszusammenschlüssen zu einer bestimmten Thematik (im Bereich der Geistesund Sozialwissenschaften wurden $26 \%$ der Labex eingerichtet) sowie »Idex« (für » initiative d'excellence ) zur Unterstützung von wissenschaftlichen Großprojekten, die pluridisziplinäre Exzellenzschwerpunkte in der universitären Lehre und Forschung hervorbringen sollten. Bei der Auswahlrunde 2011-2012 wurden acht solcher Initiativen auf der Basis von PRES finanziert, deren Kern jeweils Universitäten bildeten : Straßburg, 
Bordeaux, Toulouse und Aix-Marseille sowie vier Projekte aus dem Pariser Raum (Paris Saclay, Sorbonne Université, Université Sorbonne Paris Cité, Paris Sciences et Lettres).

Die erste Welle der Equipex (2010) hatte 52 erfolgreiche Bewerber, unter denen sich fünf Projekte aus dem Bereich der Geistes- und Sozialwissenschaften befanden, von denen wiederum zwei einen potentiellen Bezug zur Geschichtswissenschaft haben : MATRICE ${ }^{53}$, ein Projekt zur Sammlung von Zeitzeugenberichten des Zweiten Weltkriegs und der Anschläge des 11. September 2001, sowie DIME-SHS ${ }^{54}$, das sich allgemein mit der Datensammlung in den Geistes- und Sozialwissenschaften auseinandersetzt. Die zweite Welle der Equipex (2011) hatte 36 erfolgreiche Bewerber, davon fünf in den Geistes- und Sozialwissenschaften, unter denen wiederum ein Projekt in direktem Zusammenhang mit der Geschichte steht: BIBLISSIMA ${ }^{55}$ (Bibliotheca Bibliothecarum Novissima), ein Observatorium des schriftlichen Kulturerbes des Mittelalters und der Renaissance, das vom Campus Condorcet (einer erfolgreichen Initiative der "opération campus«, die darauf abzielt, mehrere wissenschaftliche Einrichtungen auf einem neuen Campus zusammenzuführen) getragen wird, welcher wiederum hauptsächlich vom PRES Hesam gebildet wird.

Auch die Einrichtung und Ausstattung der Labex verlief in zwei Wellen. In der ersten (2011), die 100 erfolgreiche Projekte umfasste, stammten 25 aus dem Bereich der Geistesund Sozialwissenschaften. Folgende Projekte haben dabei Berührungspunkte mit der Geschichte : CAP (Création, Arts et Patrimoine) ${ }^{56}$, getragen von der Universität Paris I Sorbonne-Panthéon und dem PRES Hesam, HASTEC (Histoire et Anthropologie des Savoirs, des Techniques Et des Croyances) ${ }^{57}$, getragen von der EPHE und dem PRES Hesam, LaScArBx ${ }^{58}$ (L'usage du monde par les sociétés anciennes: processus et formes d'appropriation de l'espace sur le temps long), getragen von der Universität Bordeaux, RESMED $^{59}$ (Religions et Sociétés dans le Monde Méditerranéen) getragen vom PRES ParisSorbonne, sowie TRANSFERS ${ }^{60}$ (Transferts Matériels et Culturels, Traduction, Interfaces), getragen von der ENS.

Dazu kamen in der zweiten Welle (2012) unter den 71 ausgewählten Projekten (davon 14 in den Geistes- und Sozialwissenschaften) die fünf folgenden mit einer deutlich erkennbaren historischen Ausrichtung: ARCHIMEDE ${ }^{61}$ (Archéologie et Histoire de la Méditerranée et de l'Égypte Anciennes) getragen von der Universität Montpellier, COMOD ${ }^{62}$ (Constitution de la Modernité : raison, politique, religion) getragen von der Universität Lyon, $\mathrm{EHNE}^{63}$ (Écrire une Histoire Nouvelle de l'Europe) getragen von der Universität Paris-Sorbonne IV und den Universitäten Paris I Sorbonne-Panthéon und Nantes, $\mathrm{PP}^{64}$ (Les Passés dans le Présent), u.a. getragen von der Universität Paris-Ouest-Nanterre-LaDéfense, und schließlich TEPSIS ${ }^{65}$ (Transformation de l'État, Politisation des Sociétés, Institution du Social), getragen vom PRES Hesam.

$40 \mathrm{Zu}$ erwähnen ist außerdem das Labex RFIEA+ (Réseau Français des Instituts d'Études Avancées Plus), das zwar nicht explizit disziplinär ausgerichtet ist, sondern die bereits genannten Instituts d'Études Avancés (IEA) in Lyon, Nantes, Paris und Aix-Marseille verbindet. Seine Tätigkeit zielt auf eine interdisziplinäre öffnung und Internationalisierung der Geistes- und Sozialwissenschaften und auf die Erforschung übergreifender Themen wie Sprache, komplexe Systeme, die Organisation von Gesellschaften oder das gemeinsame Vorgehen im Austausch zwischen Geistes- und Sozialwissenschaften und anderen Disziplinen. Die Geschichtswissenschaft findet hier also uneingeschränkt ihren Platz in einem reichhaltigen Spektrum aus Ausbildungsmodulen, wissenschaftlichen veranstaltungen und 
Gastwissenschaftlerprogrammen mit thematischer Ausrichtung etwa auf die Gesellschaft, die Sprache, die Erinnerung oder die kulturelle und soziale Vielfalt.

Insgesamt gibt es also zehn Labex mit einem deutlichen geschichtswissenschaftlichen Profil, deren Aufgabe es in den nächsten zehn Jahren sein wird, die Forschung zu strukturieren und zu bündeln. Sieben von ihnen sind in und um Paris angesiedelt, drei verfolgen einen kulturellen Ansatz und für drei weitere steht der Mittelmeerraum im Zentrum der Forschungsaktivitäten.

3. Die dritte wichtige Entwicklung bestand in der ausschließlich von und für die Universitäten neu gefassten Strukturierung der Doktorandenausbildung innerhalb von Doktorandenschulen (écoles doctorales, ED), die zu Recht als der Ort einer Heranführung an die Forschung durch die Forschung angesehen werden. Seit 2006 hat sich das Modell solcher Doktorandenschulen, die bereits seit 2000 in großer Zahl entstanden waren, allgemein durchgesetzt. Ihre Aufgabe ist es, Doktoranden einen strukturierten Rahmen für die Promotion zu bieten, die Promotionsstipendien und Arbeitsverträge für Doktoranden zu verwalten, spezifische Ausbildungsmodule $\mathrm{zu}$ offerieren, sich um die Berufseingliederung von Doktoranden und frisch Promovierten zu kümmern, aber auch Publikationsmöglichkeiten für die Ergebnisse der laufenden Forschungsarbeiten anzubieten (wie die Zeitschrift »Hypothèses« der Doktorandenschule in Geschichte der Universität Paris 1 Panthéon-Sorbonne ${ }^{66}$ ).

43 Alle Doktorandenschulen müssen vom Ministerium anerkannt ( akkreditiert «) werden ${ }^{67}$ und unterliegen regelmäßigen Evaluierungen durch die Agence d'Évaluation de la Recherche et de l'Enseignement Supérieur (AERES). Sie versammeln und verbinden um die Promotion herum eine Großzahl von Forschergruppen, Forschungszentren und spezifischen Doktorandenstudiengängen. Momentan gibt es, alle Disziplinen eingeschlossen und über alle einschlägigen Universitäten und Hochschuleinrichtungen hinweg, 286 Doktorandenschulen ${ }^{68}$. Das Feld der Geschichtswissenschaften verteilt sich dabei auf 26 écoles doctorales, die sich mit Vor- und Frühgeschichte befassen, 65 Doktorandenschulen, die sich mit Geschichte und Archäologie der antiken und mittelalterlichen Welt beschäftigen, und 76 Strukturen, die der frühneuzeitlichen und neueren Geschichte gewidmet sind ${ }^{69}$.

Zur Koordination dieser zahlreichen Reformen und Initiativen hat das französische Hochschulministerium zudem zwei Agenturen gegründet, die heute zentrale Akteure der französischen Forschungspolitik sind : zum einen die Agence Nationale de la Recherche $(\mathrm{ANR})^{70}$, die 2005 ins Leben gerufen wurde und zur Aufgabe hat, die öffentliche Forschung und Forschungsprojekte in Partnerschaft vor allem durch die Ausschreibung von Projekten mit zeitlich befristeter Dauer $\mathrm{zu}$ finanzieren. Ihre kompetitiven Projektausschreibungen ( »appels à projet compétitifs«) können thematisch ausgerichtet oder offen sein (dann spricht man von einer »weißen Ausschreibung", »appel blanc«).

Gemeinsam mit der DFG schreibt die ANR das auf die Geistes- und Sozialwissenschaften beschränkte deutsch-französische Programm aus ${ }^{71}$. Die Liste der Themen der im Rahmen dieses Programms geförderten Projekte ist ein guter Indikator für die aktuellen Forschungstendenzen in beiden Ländern. Im Bereich der Geschichte waren dies 2013 folgende Vorhaben : »Politische Korruption: Praktiken der Begünstigung und öffentliche Debatten in Deutschland und Frankreich (19.-20. Jahrhundert)«, »Archäologie einer Transformationszeit. Das Gräberfeld von Niedernai und das 5. Jahrhundert am Oberrhein« und »Literarische Kreativität und biologisches Wissen im 19. Jahrhundert« . Wie aus einer Zwischenbilanz der Jahre 2007-2012 hervorgeht, hatten 36 der insgesamt 
113 geförderten Projekte des Programms (also fast ein Drittel) einen geschichtswissenschaftlichen Bezug, darunter 17 Projekte in Archäologie, 16 in Geschichte (im engeren Sinne) und drei in Kunstgeschichte. Konzipiert ist die ANR als eine Forschungsförderungseinrichtung, die die parallele Finanzierung durch das CNRS, die sich vor allem auf die Grundausstattung richtet, begleiten und ergänzen soll.

Die zweite vom Ministerium gegründete Institution ist die Evaluationsagentur Agence d'Évaluation de la Recherche et de l'Enseignement Supérieur (AERES) ${ }^{72}$, die ebenfalls 2006 ins Leben gerufen wurde und 2007 ihre Arbeit aufgenommen hat. Ihre Aufgabe ist es, geförderte Projekte zu evaluieren - sie ist also nur die logische Ergänzung in einem System der Forschungsförderung durch Projektfinanzierung. Daneben evaluiert sie aber auch Universitäten und Hochschuleinrichtungen, Forschungsorganisationen und -institute sowie Studiengänge und Abschlüsse im gesamten Hochschulsektor. Im Jahr 2013 waren an den Evaluationsverfahren 4.000 Experten beteiligt, die 39 Universitäten, 600 Forschungsstudiengänge, 492 Abschlüsse, zwölf Doktorandenschulen und zwei Forschungsorganisationen begutachteten. Im Juli 2013 wurde beschlossen, die AERES durch den Haut Conseil de l'Évaluation de la Recherche et de l'Enseignement Supérieur (HCERES) zu ersetzen.

Von diesen Veränderungen abgesehen, war die Einrichtung dieser beiden Agenturen Teil einer größeren Bewegung, deren Ziel es ist, die Forschungskapazitäten der Universitäten auszubauen. Geschehen soll dies im Rahmen der Empfehlungen des sogenannten LRUGesetzes (Loi Relative aux libertés et responsabilités des Universités), das 2007 verabschiedet wurde und die Autonomie und Attraktivität der Universitäten stärken soll. Im Juli 2013 wurde dieses Gesetz unter dem Namen »Loi pour l'Enseignement Supérieur et la Recherche (LESR) neu gefasst ${ }^{73}$. Das stärkere Engagement der Universitäten im Forschungsbereich zeigt sich im Übrigen auch in der starken Stellung, die universitäre Einrichtungen in den Forschungs- und Nachwuchsförderungsprogrammen der DeutschFranzösischen Hochschule (DFH) haben - vor zehn Jahren wäre ihr Platz dort noch anders ausgefallen $^{74}$.

Die DFH wurde 1997 gegründet und hat 1999 ihre Arbeit aufgenommen. Ihr erstes Ziel war die Entwicklung und der Ausbau integrierter, bi-nationaler und bilingualer deutschfranzösischer Studiengänge in allen Disziplinen. Heute existieren 153 solcher Studiengänge, die je zu einem Drittel auf die Ingenieurwissenschaften, die Rechts- und Verwaltungswissenschaften und die Geistes- und Sozialwissenschaften entfallen und etwa 6.200 Studierende umfassen, die einen Teil ihrer Ausbildung im Partnerland absolvieren und ein Doppeldiplom vorbereiten ${ }^{75}$. Die Abteilung Forschungsförderung der DFH unterstützt daneben 23 Deutsch-Französische Doktorandenkollegs, von denen neun geistes- und sozialwissenschaftlich und davon wiederum sechs speziell geschichtswissenschaftlich ausgerichtet sind. Auf französischer Seite kooperieren dabei fünf Universitäten (Paris I Panthéon-Sorbonne, Paris Diderot-Paris VII, Dijon, AixMarseille und Straßburg) und nur ein grand établissement (die EHESS) mit deutschen Hochschulen. In solchen Entwicklungen kann man das Ergebnis einer entschiedenen Politik seitens der französischen Doktorandenschulen sehen (die EHESS und Paris I Panthéon-Sorbonne sind die zwei meistbesuchten Doktorandenschulen im Bereich der Geschichtswissenschaft in Frankreich), die Ausdruck ist für eine Gewichtsverschiebung der Nachwuchsförderung zugunsten der Universitäten.

Im Vergleich zur Situation in Deutschland können sich in Frankreich Professoren und maîtres de conférences, die sich $\mathrm{zu}$ Forschungszwecken eine Zeitlang von ihren 
Verpflichtungen in der Lehre, der universitären Selbstverwaltung und der Vorbereitung auf die nationalen Auswahlverfahren befreien lassen wollen, nicht auf Assistenten oder Lehrbeauftragte stützen. Auch haben sie nur in geringerem Maße das Anrecht auf Forschungsfreisemester und können kaum Forschungsaufenthalte in Forschungsorganisationen wie den deutschen Max-Planck-Instituten wahrnehmen. Es gibt jedoch zwei Programme, die ihnen im Rahmen ihrer Universitätskarriere Freiräume für die Forschung schaffen sollen: zum einen die Delegation ans CNRS, die es einem Hochschullehrer erlaubt, bei Fortführung seiner Universitätsbezüge zwischen sechs Monate und einem Jahr an einem Forschungszentrum oder einer UMR des CNRS zu verbringen; zum anderen - und das ist die begehrtere Möglichkeit - die Teilnahme an einem Förderprogramm des Institut Universitaire de France (IUF) ${ }^{76}$. Dieses ermöglicht über alle Disziplinen hinweg - 70 maitres de conférences (Kategorie »junior «) und 38 Professoren (Kategorie »senior«) während eines Zeitraums von 5 Jahren (der für Professoren einmal verlängert werden kann, aktuell zwei Fälle) von zwei Dritteln ihrer Lehrverpflichtungen befreit zu werden und über ein jährliches Forschungsbudget von 15.000 Euro für Projekte und Publikationen zu verfügen. Im Durchschnitt (und in Abhängigkeit von der Bewerberlage) entfallen zwischen zwei und vier dieser Plätze pro Kategorie und Jahr auf Historiker.

\section{Fortdauer und Konkurrenz zweier Welten}

Der Wille, die Forschungskapazitäten der Universitäten - und zwar ab dem Promotionsniveau - auszubauen, ist also unübersehbar. Dennoch hat diese Entwicklung noch nicht dazu beigetragen, Macht und Einfluss einiger großer Hochschuleinrichtungen aus dem Bereich der grandes écoles und der grands établissements einzuschränken Institutionen, deren Funktionsweisen, Statuten, Finanzierung, Personal, Dienstverpflichtungen und Einstellungspolitik anderen Regeln als jenen der Universitäten gehorchen. Für den Bereich der Geschichtswissenschaft reicht es, die Écoles Normales Supérieures ${ }^{77}$, die École des Chartes $^{78}$, die École Pratique des Hautes Études und die École des Hautes Études en Sciences Sociales anzuführen, um sich dies bewusst zu machen.

Die an diesen Einrichtungen festangestellten Hochschullehrer tragen nicht immer den Titel eines Professors, sondern beispielsweise den Titel »directeur d'études« oder »directeur de recherches« und haben einen von den Universitätsmitarbeitern gesonderten Beamtenstatus, der sich u.a. darin äußert, dass sie nicht den gleichen Lehrverpflichtungen unterliegen. Auch haben diese Einrichtungen eigene Auswahlverfahren für ihre Studenten (entweder auf der Grundlage von Bewerbungsunterlagen oder eines gesonderten Aufnahmeverfahrens), d.h. die Einschreibung in diesen Hochschulen ist kein Recht wie an den Universitäten. In die Liste dieser Einrichtungen gehören darüber hinaus auch andere prestigereiche Institutionen wie die École Polytechnique (gegründet 1794$)^{79}$ und die École Nationale des Ponts et Chaussées (gegründet 1747) ${ }^{80}$, die ebenfalls über Forschungsprogramme zur Geschichte der Technik, der Ingenieurswissenschaften oder den internationalen Beziehungen verfügen.

Grundlage der Forschungsaktivitäten in diesen grands établissements ist vor allem die Lehrform des Seminars und die Philosophie einer Ausbildung zur Forschung durch die Forschung (»formation à la recherche par la recherche«). Transversale, übergreifende 
und interdisziplinäre Fragestellungen finden dabei vor allem an der EHESS und der EPHE einen Platz ${ }^{81}$, die wenig oder gar nicht in die Vorbereitung auf die nationalen Auswahlverfahren eingebunden sind, von hohen Studentenzahlen und der damit einhergehenden Belastung durch die Korrektur von Klausuren und Hausarbeiten verschont bleiben und nicht den traditionellen Abgrenzungsbestrebungen zwischen Perioden und Fakultäten unterliegen. Ihren Forschern bieten diese Institutionen eine erhebliche Freiheit und die Möglichkeit einer hohen Mobilität, vor allem auf internationaler Ebene. Über solche Arbeits- und Forschungsbedingungen verfügen Universitätsangehörige nicht oder nicht in gleichem Maße.

Ermöglicht wird diesen Einrichtungen dadurch, Forschungszentren oder -strukturen zu schaffen, die sich mit einem spezifischen Kulturraum auseinandersetzen oder sich für bestimmte bilaterale oder internationale Beziehungen interessieren. Im deutschfranzösischen Bereich wäre hier besonders auf das Centre Interdisciplinaire d'Études et de Recherches sur l'Allemagne (CIERA) ${ }^{82}$ hinzuweisen, das 1997 gegründet und 2001 in der Rechtsform eines Groupement d'Intêret Public (GIP) eingerichtet wurde. Dieses Zentrum gehört nicht zur EHESS, da es die Forschungs-, Ausbildungs-, Mobilitäts-, Betreuungs-, Veröffentlichungs- und Lehraktivitäten von elf Mitgliedsinstitutionen (darunter fünf Universitäten in Paris, Lyon und Straßburg) bündelt, die sich im Rahmen eines interdisziplinären Ansatzes mit der deutschsprachigen Welt beschäftigen. Es stützt sich aber auf die Ressourcen mehrerer außeruniversitärer Hochschuleinrichtungen: der Écoles Normales Supérieures in Paris und Lyon, der EHESS (hier vor allem die UMR 8131 Centre Georg Simmel - Recherches franco-allemandes en sciences sociales), aber auch der Fondation Maison des Sciences de l'Homme in Paris und Sciences Politiques Grenoble (die Aufnahme von Sciences Po Paris in das Netzwerk steht bevor).

Komplettieren lässt sich das Mosaik von außeruniversitären Einrichtungen bzw. Hochschulen, die im Vergleich zu den Universitäten über einen abweichenden Status verfügen, noch durch einige andere Einrichtungen, an denen ebenfalls historische Forschungen betrieben werden. Darunter fällt das INALCO ${ }^{83}$ (Institut National des Langues et Civilisations Orientales, kurz auch »Langues O« genannt), das auf eine erste Gründung im Jahr 1669 zurückgeht und 1795 neu gegründet wurde. An dieser Hochschule werden vor allem nicht westliche und außereuropäische - also vor allem afrikanische, indoamerikanische, west-, zentral- und ostasiatische, aber auch russische, eurasische und zentraleuropäische - Sprachen und Kulturen gelehrt. Ähnlich dem Institut d'Études Politiques (IEP oder auch Sciences Po) ${ }^{84}$ handelt es sich auch beim INALCO um ein grand établissement, also um eine universitätsnahe, aber nicht universitätsgleiche Institution, die ihre eigenen Abschlüsse vergibt, Forschungszentren und Spezialbibliotheken unterhält und Publikationen veröffentlicht.

Das IEP wurde 1872 als Stiftung gegründet und 1969 in eine öffentliche Einrichtung umgewandelt (EPCSCP, siehe oben). Auch diese Hochschule verfügt über ihre eigenen Abschlüsse, ihre Rekrutierungsverfahren für Studenten und Hochschullehrer ${ }^{85}$, ihre Bibliothek und Doktorandenschule sowie über Zweigstellen in den Regionen (sechs an der Zahl, in Reims, Dijon, Le Havre, Menton, Nancy und Poitiers). Daneben gehören zu ihr eine eigene nationale Stiftung für Politikwissenschaften (Fondation Nationale des Sciences Politiques, FNSP) ${ }^{86}$ sowie eine Reihe von Forschungszentren, darunter das CERI (Centre d'Études et de Recherches Internationales, UMR 7050) ${ }^{87}$, das Centre d'Histoire de Sciences Po (CHSP) ${ }^{88}$ und das Centre d'Études de la Vie Politique Française (CEVIPOF, UMR 7048, jetzt umbenannt in Centre de Recherches Politiques de Sciences Po) ${ }^{89}$. Darüber 
hinaus existieren neben dem Pariser »Original« zehn weitere Instituts d'Études Politiques, die mehr oder weniger nach dem gleichen Modell einer unabhängigen Lehranstalt für Politikwissenschaften geschaffen wurden; acht unter ihnen sind inzwischen einer Universität angegliedert (Straßburg, Toulouse, Grenoble, Lyon, Bordeaux, Aix-en-Provence, Lille und Rennes).

Erwähnt werden müssen schließlich auch noch die französischen grandes écoles im Ausland. Sie sind den deutschen geisteswissenschaftlichen Instituten im Ausland vergleichbar, die inzwischen unter dem Dach der Max Weber Stiftung zusammengefasst worden sind ${ }^{90}$. Es handelt sich um die drei Écoles Françaises à l'Étranger (EFE) in Madrid, Rom und Athen (daneben gehören zu den EFE Einrichtungen in Kairo und in Süd-OstAsien). Die École Française de Rome ${ }^{91}$ hat eine besonders lange Tradition, denn ihre Anfänge reichen bis ins Jahr 1829 zurück. 1875 wurde sie als Auslandshochschule neu gegründet, steht seit diesem Zeitpunkt unter der Aufsicht der Académie des Inscriptions et des Belles Lettres und ist dem Hochschulministerium unterstellt. Sie bietet auf drei Jahre befristete Forschungsstellen an, organisiert Künstleraufenthalte und beherbergt neben Archäologen auch zahlreiche Stipendiaten. Die Forschungsprogramme, wissenschaftlichen Veranstaltungen und Veröffentlichungen setzen den Schwerpunkt zwar auf die italienische bzw. französisch-italienische Geschichte, öffnen sich zunehmend aber einer gesamteuropäischen Perspektive. Dies gilt ebenso für die École Française d'Athènes (gegründet 1846) ${ }^{92}$ und die in Madrid beheimatete »Casa de Velasquez «(gegründet 1929) ${ }^{93}$. Alle diese Institutionen bieten zwar Aufenthalte für Stipendiaten, Nachwuchswissenschaftler und gestandene Forscher an, aber verleihen nicht den Doktorgrad. Die Arbeits- und Forschungsbedingungen sind jedoch ähnlich vorteilhaft wie in etlichen der eben erwähnten außeruniversitären Instituten, Einrichtungen und Hochschulen.

Auf die eine oder andere Weise ist der Gegensatz zwischen außeruniversitärer und universitärer Forschung in Frankreich also nach wie vor präsent. Hier und da schafft er Konkurrenzsituationen und Missverständnisse. Und mitunter trägt er sogar schizophrene Züge, denn auf dem Gebiet der Hochschulbildung ist die französische Gesellschaft einerseits tief einem unbarmherzigen Elitismus verbunden (wie er sich etwa im System der Vorbereitungsklassen äußert) und hängt andererseits am Wert einer weitestgehenden Chancengleichheit (Recht auf einen Studienplatz für jeden Abiturienten, ohne jeden Numerus clausus) $\mathrm{an}^{94}$.

\section{Forschung außerhalb der Universitäten und Hochschulen}

Ein Teil der Forschung in Frankreich (Tagungen, Veröffentlichungen, Teilnahme an europäischen Projekten) wird von Vereinigungen und Vereinen organisiert, in denen sich Historiker und Forscher nach Perioden zusammengefunden haben. Im Unterschied zu Deutschland gibt es hier keinen Historikerverband ${ }^{95}$, der alle zwei Jahre gemeinsam mit dem Verband der Geschichtslehrer ${ }^{96}$ den Historikertag ${ }^{97}$ organisiert und also alle Lehrenden aus Sekundarstufe und Hochschule in einer großen, den gesamten Berufszweig umfassenden Veranstaltung zusammenbringt. Aber auch Frankreich hat eine einzigartige Veranstaltung von landesweiter Ausstrahlung aufzuweisen, die in Deutschland fehlt und jedes Jahr einige Tage lang die Gesamtheit der historischen »Zunft« zusammenführt, also Hochschullehrer und Forscher, aber auch 
Schüler und Studenten sowie Verlage und andere Medien. Es handelt sich um das seit 1998 veranstaltete »Rendez-vous de l'histoire« in Blois ${ }^{98}$, das jährlich mehr als 25.000 Besucher in über 300 Veranstaltungen zusammenbringt (ein deutsches Pendant existiert in Weimar, der Partnerstadt von Blois, das 2013 zum fünften Mal stattgefunden hat) ${ }^{99}$.

59 Außerhalb dieses Ereignisses gibt es in Frankreich aber keine gemeinsamen Strukturen für Historiker aus dem Schul- und Hochschulbereich. Für Lehrer in der Sekundarstufe findet man einerseits die Association des professeurs d'histoire et de géographie (APHG) ${ }^{100}$, die eine Zeitschrift herausgibt und sich als Standesvertretung um den Status des Lehrerberufs, die Einstellungspolitik und die Lehrpläne in Geschichte in den collèges und lycées ${ }^{101}$ kümmert. Doch die Kontakte zwischen der APHG und der universitären Sphäre sind spärlich und wenig strukturiert.

60 Für Universitätshistoriker sind mehrere Verbände zuständig. In der Alten Geschichte ist dies die Société des Professeurs d'Histoire Ancienne à l'Université (SOPHAU) ${ }^{102}$, die 1958 gegründet wurde und aktuell 400 Hochschullehrer als Mitglieder zählt, Tagungen organisiert und ein Mitgliederverzeichnis herausgibt. Im Bereich der Mediävistik spielt die Société des Historiens Médiévistes de l'Enseignement Supérieur Public (SHMESP) ${ }^{103}$ eine ähnliche Rolle. Sie wurde 1969 gegründet und hat aktuell 650 Mitglieder. Anders als die SOPHAU tritt sie jedoch stärker durch eigene Publikationen hervor (vor allem durch die Veröffentlichung der bislang 43 Tagungsbände ihrer Jahrestreffen, die heute vielfach im Ausland stattfinden) und durch das Angebot eines fachwissenschaftlichen Informationsdienstes. Auch die Association des Historiens Modernistes des Universités Françaises (AHMUF) verzeichnet ähnliche Aktivitäten. Sie wurde 1967 gegründet und deckt die Frühe Neuzeit ab (16.-18. Jahrhundert) ${ }^{104}$. Im Bereich der neueren und Zeitgeschichte schließlich ist die Association des Historiens Contemporanéistes de l'Enseignement Supérieur et de la Recherche (AHCESR) ${ }^{105}$ tätig, die 1965 gegründet wurde.

61 Alle vier Verbände sind wichtige Orte des fachwissenschaftlichen Austausches und bieten die Möglichkeit, sich schnell und bequem über die Namen, Funktionen und Heimatinstitutionen französischer Historiker $\mathrm{zu}$ informieren. Daneben lassen sich anhand der verbandseigenen Publikationen und Veranstaltungen die neuesten Tendenzen der historischen Forschung in Frankreich verfolgen. Ihnen zur Seite steht darüber hinaus die 1967 gegründete Association des Germanistes de l'Enseignement Supérieur (AGES) ${ }^{106}$, deren Veranstaltungen und Veröffentlichungen ebenfalls Teil der französischen historischen Forschung zur deutschsprachigen Welt sind ${ }^{107}$. Denn zahlreiche Germanisten, die sich mit der deutschen Literatur und Kultur beschäftigen, führen in enger Kooperation mit Historikern Forschungs- und Publikationsprojekte durch, die eine klare geschichtswissenschaftliche Ausrichtung haben - und zwar in deutlich größeren Proportionen, als sich dies im Vergleich mit romanistischen Lehrstühlen und Instituten in Deutschland beobachten lässt.

62 Vergessen werden sollte auch das Comité des Travaux Historiques et Scientifiques (CTHS) nicht ${ }^{108}$, das eine zentrale Rolle für die historische Forschung spielt. Es wurde im Jahr 1834 von François Guizot gegründet, um große Akteneditionen zur Geschichte Frankreichs vorzubereiten, und ist seit 2007 Teil der École des Chartes. Seine derzeit neun geschichtswissenschaftlichen Arbeitsbereiche haben die Aufgabe, sozialwissenschaftliche Forschungsprojekte und Publikationen zu Frankreich und seinen Regionen zu fördern, geschichtswissenschaftliche Forschungsergebnisse einem breiteren Publikum näherzubringen und Texte, Verzeichnisse, Forschungsleitfäden, Datenbanken und Arbeitshilfsmittel zu veröffentlichen. Die Jahrestagung des CTHS führt zudem die 
Arbeitsergebnisse und Projekte zahlreicher gelehrter Gesellschaften und ihrer Untergliederungen zusammen ${ }^{109}$.

Die historische Forschung findet in Frankreich Unterstützung und ein Echo aber auch durch Institutionen, die man so weder in Deutschland noch in anderen Ländern findet. An erster Stelle dieser Einrichtungen steht das 1530 eingerichtete Collège de France ${ }^{110}$. Unter seinen gegenwärtigen Lehrstühlen nimmt die Geschichte einen prominenten Platz ein : Alte Geschichte, Geschichte Mesopotamiens, altägyptische Geschichte, vorislamische Geschichte Zentralasiens, griechische Geschichte, arabische Geschichte, chinesische Geschichte, Geschichte des antiken Roms, Geschichte Japans, Geschichte der mittelalterlichen Philosophie, mittelalterliche Literaturgeschichte, Geschichte von Schrift und Schriftlichkeit ... und zuletzt ein Lehrstuhl für Globalgeschichte der Frühen Neuzeit der erste seiner Art, besetzt mit Sanjay Subrahmanyam. Die zweite altehrwürdige Institution, die hier zu nennen ist, ist die Académie des Inscriptions et Belles Lettres $(\text { AIBL })^{111}$, die im Jahr 1663 gegründet wurde und eine der fünf Akademien des Institut de France bildet. Durch Preise, Veröffentlichungen, eine Zeitschrift (»Le Journal des Savants«), Ausgrabungsstätten, die oben erläuterten französischen Auslandsinstitute, Atlanten und Quellensammlungen ist die AIBL ein zentraler Akteur bei der Förderung und Unterstützung der historischen und archäologischen Forschung in Frankreich.

\section{Schluss}

Dem nicht französischen Leser dieses ein wenig barocken Überblicks über die Strukturen und Institutionen der historischen Forschung in Frankreich wird sich ohne Zweifel der Eindruck aufdrängen, dass sich Strukturen und Kompetenzen hier allenthalben überlagern und die Architektur dieser Überlagerungen durch einen systemischen Gegensatz bestimmt wird: Trotz aller Reformversuche fallen die Forschungsaktivitäten an den Universitäten und an den außeruniversitären Einrichtungen weiterhin auseinander und setzt sich die Region Paris, wo die Hälfte der Universitätshistoriker lehrt oder wohnt, vom Rest des Landes ab. Zwischen beiden Blöcken sind in den letzten Jahren zwar neue Verbindungen entstanden : Die jüngsten Reformen - wahrscheinlich die tiefgreifendsten, die es im Hochschulbereich seit 1945 gegeben hat - haben neue Rahmenbedingungen geschaffen und die Linien verschoben, trotz eines von zunehmenden Einschränkungen geprägten finanziellen Kontextes. Doch im Großen und Ganzen ist die französische Forschungslandschaft die gleiche geblieben. Gesteuert wird sie nach wie vor von zentraler Stelle, was in Zeiten knapper Haushalte einen Rückgang staatlicher Förderung zur Folge hat, der nicht durch subsidiäre Strukturen der Wissenschaftsförderung aufgefangen wird, die - wie die deutschen Stiftungen oder Wissenschaftsorganisationen ausgleichend eingreifen und etwa $\mathrm{zu}$ einem erheblichen Teil die Stellen von Universitätsangehörigen finanzieren können. So weit ist es in Frankreich (noch ?) nicht. Dennoch lassen die jüngsten Entwicklungen im Bereich von Forschung und Lehre eine Annäherung zwischen Universitäten und grandes écoles erkennen. PRES (bzw. ComUEs) und andere Forschungsverbände sind neue föderative Strukturen, die ihre Mittel über einen Prozess der Vertragsförderung und der Evaluation erhalten und sich insofern international üblichen Praktiken annähern. Damit greift langsam auch bei den Forschungsstrukturen eine Internationalisierung um sich, die auf der Ebene der Forscher und ihrer Ideen längst Alltag ist, sich bislang aber oft noch in spezifischen und für 
Außenstehende nur schwer verständlichen Verfahren und Praktiken vollzieht, die noch dazu permanenten Veränderungen unterworfen sind.

\section{NOTES}

1. Die deutsche Ausgabe «100 Schlüsselbegriffe für Deutsche und Franzosen« erschien 1989 bei Piper in München, die französische 1990 unter dem Titel »Au jardin des malentendus« bei Actes Sud in Paris.

2. Verfasst von Hansgerd Schulte, S. 344-347 der französischen Ausgabe.

3. Falk Bretschneider, Bernd Klesmann, Rahul Markovits, Universités, instituts de recherche extra-universitaires et associations, in: Falk Bretschneider, Mareike König (Hg.), Faire de l'histoire en Allemagne. Un guide pour les jeunes chercheurs français : http:// www.perspectivia.net/content/publikationen/scholar-guide/histoire-en-allemagne/ bretschneider-klesmann-markovits_structures. Alle Links wurden am 2.7.2014 überprüft.

4. Die Zahlen stützen sich auf den letzten Zustandsbericht des Ministeriums für Hochschulbildung und Forschung, Nr.6 Februar 2013, http://www.enseignementsuprecherche.gouv.fr/cid66659/l-etat-de-l-enseignement-superieur-et-de-la-recherche-n-6-

fevrier-2013.html.

5. CUEs sind EPCSCPs (Établissements Publics à Caractère Scientifique, Culturel et Professionnel), die nach dem Gesetz vom 22 Juli 2013 die PRES ersetzen sollen, deren Status als Établissement Public de Coopération Scientifique (EPCS) bezeichnet wurde. 2014 wurden 7 CUE eingerichtet: Aquitaine (um die Universität von Bordeaux), Universität Grenoble, Universität Lyon, Universität Paris-Est, Universität Paris-Saclay, Universität Toulouse, Hesam (um die EPHE, EHESS, Paris I und die École des Arts et Métiers.)

6. »Programme Cadre de Recherche et Développement 2007-2013«, das 8. Programm für den Zeitraum 2014-2020 trägt den Namen »Horizon 2020«.

7. MESR, http://www.enseignementsup-recherche.gouv.fr/.

8. Siehe dazu auch den Beitrag von Claire Gantet "Zwischen französischen Besonderheiten und internationaler Öffnung : Unterricht und Lehrerausbildung in Frankreich« in diesem Wegweiser ( http://ifha.revues.org/7857).

9. EHESS, http://www.ehess.fr/fr/.

10. EPHE, http://www.ephe.sorbonne.fr/. Die EHESS, die EPHE, die Universität Paris I PanthéonSorbonne, die École des Chartes, die École Française d'Extrême-Orient, das Institut National des Études Démographiques, das Institut National d'Histoire de l'Art und einige andere Einrichtungen formen seit 2010 den PRES Hésam (Hautes Études Arts et Métiers). Dieser soll in den nächsten Jahren im Norden von Paris den neuen Campus Condorcet beziehen und zu einem der wichtigsten geistes- und sozialwissenschaftlichen Zentren Europas werden.

11. CNRS, http://www.cnrs.fr/.

12. INED, http://www.ined.fr/.

13. Emmanuelle Picard, Enseignement supérieur et recherche, in: Christian Delacroix, François Dosse, Patrick Garcia, Nicolas Offenstadt (Hg.), Historiographies. Concepts et débats, Paris 2010, Bd. 1, S. 141-152. Christian Delacroix, François Dosse, Patrick Garcia, Les courants historiques en France. $\mathrm{XIX}^{\mathrm{e}}-\mathrm{XX}^{\mathrm{e}}$ siècle, Paris 2005 (2. Auflage). Antoine Prost, Douze leçons sur l'histoire, Paris 1996. Olivier Dumoulin, Le rôle social de l'historien, Paris 2003. 
14. ENS, http://www.ens.fr/.

15. ENC, http://www.enc.sorbonne.fr.

16. EPHE, http://www.ephe.sorbonne.fr/.

17. Gérard Noiriel, Sur la » crise« de l'histoire, Paris 1996.

18. IRHT, http://www.irht.cnrs.fr/.

19. IHTP, http://www.ihtp.cnrs.fr/.

20. Diese Verteilung entspricht der Konzentration von Wissenschaftlern in der Germanistik bzw. der deutschen Geschichte, wie sie vom CIERA in seiner Datenbank von 1.150 Wissenschaftlern in 317 französischen Forschungszentren zur deutschsprachigen Welt zusammengefasst werden. Paris 2004, jetzt mit Karte des Netzwerks online zugänglich unter: http://www.ciera.fr/ repertoire/web/aide.html.

21. Gérard Noiriel, Métier/communauté, in: Christian Delacroix, François Dosse, Patrick Garcia, Nicolas Offenstadt (Hg.), Historiographies. Concepts et débats, Paris 2010, Bd. 1, S. 518-528.

22. Vgl. die Liste der Sonderforschungsbereiche, die von der Deutschen Forschungsgemeinschaft in den Geisteswissenschaften finanziert werden: http://www.dfg.de/foerderung/programme/ listen/index.jsp?id=SFB.

23. Institut catholique de Lille, http://www.univ-catholille.fr/.

24. Institut catholique Paris, http://www.icp.fr/.

25. Institut catholique Lyon, http://www.univ-catholyon.fr/.

26. 34 dieser UMR widmen sich der Antike und dem Mittelalter: https://web-ast.dsi.cnrs.fr/l3c/ owa/annuaire.recherche/index.html. Genauso viele beschäftigen sich mit der Frühen Neuzeit, der neueren und neusten Geschichte: https://web-ast.dsi.cnrs.fr/l3c/owa/annuaire.recherche/ index.html.

27. UMR 8558, http://crh.ehess.fr/.

28. UMR 8589, http://lamop.univ-paris1.fr/. An der Universität Paris I Panthéon-Sorbonne auch die Antike mit dem UMR 8210 Anhima, Anthropologie et Histoire des Mondes Antiques, http:// anhima.fr/.

29. Vgl. das Centre Roland Mousnier UMR 8596 der Universität Paris-Sorbonne IV, http:// www.rolandmousnier.cnrs.fr/.

30. Vgl. das Institut d'Histoire Moderne et Contemporaine (IHMC), UMR der ENS Paris, http:// www.ihmc.ens.fr/.

31. Vgl. beispielsweise die UMR 6298 der Universität Bourgogne, http://www.artehis-cnrs.fr/. Vgl. auch das CIHAM der Universität Lyon, http://ciham.ish-lyon.cnrs.fr/. Vgl. auch das Centre Michel de Boüard der Universität Caen (UMR 6273, http://www.unicaen.fr/crahm/), AOROC UMR 8546 der ENS Paris, http://www.archeo.ens.fr/.

32. Vgl. beispielsweise den Platz der Historiker in der UMR 5600 "Environnement, ville, société« in Lyon, http://umr5600.ish-lyon.cnrs.fr/, der UMR 7324 "Cités, Territoires, Environnement et Sociétés» der Universität Tours: http://citeres.univ-tours.fr/ oder der UMR 8185 „Espaces, Nature et Culture« der Universität Paris-Sorbonne IV, http://www.enec.cnrs.fr/spip.php? article79.

33. Vgl. das Centre Alexandre Koyré UMR 8560 zur Wissenschafts- und Technikgeschichte: http://www.koyre.cnrs.fr/\#\&panel1-1.

34. Vgl. das Centre André Chastel UMR 8150 der Universität Paris-Sorbonne IV, http:// www.centrechastel.paris-sorbonne.fr

35. Jean-François Sirinelli, Pascal Cauchy, Claude Gauvard (Hg.), Les historiens français à l'œuvre 1995-2010, Paris 2010 und Christophe Granger (Hg.), À quoi pensent les historiens? Faire de l'histoire au XXI ${ }^{\mathrm{e}}$ siècle, Paris 2013.

36. Zu Spanien vgl. die UMR 5136 Framespa der Universität Toulouse, http://framespa.univtlse2.fr/. 
37. Centre Georg Simmel der EHESS, UMR 8131, http://centregeorgsimmel.ehess.fr/.

38. Vgl die UMR 8167 »Orient et Méditerranée« der Universitäten Paris I Panthéon-Sorbonne und Paris-Sorbonne IV und der EPHE, http://www.orient-mediterranee.com/spip.php? rubrique101\&lang=fr, oder die UMR 7303, 7297 et 7299 der Universität Aix-en-Provence/ Marseille, http://telemme.mmsh.univ-aix.fr, http://www.mmsh.univ-aix.fr/cpaf/ und http:// ccj.cnrs.fr/ (Centre Camille Julian).

39. Vgl. das Centre Chine-Corée-Japon UMR 8173, http://www.ehess.fr/centres/cecmc/ und die UMR 8170 des Centre Asie du Sud-Est der EHESS: http://case.vjf.cnrs.fr/.

40. Réseaux thématiques de recherche avancée et de recherche et de soins, http:// www.enseignementsup-recherche.gouv.fr/cid56330/les-reseaux-thematiques-de-recherchesavancees-et-de-recherche-et-de-soins.html.

41. École d'Économie de Toulouse, http://www.tse-fr.eu/index.php.

42. École d'Économie de Paris, http://www.parisschoolofeconomics.eu/.

43. Réseau français des Instituts d'études avancées, http://www.cnrs.fr/inshs/recherche/ rfiea.htm.

44. Collegium de Lyon, http://www.collegium-lyon.fr/.

45. Institut Méditerranéen des Recherches Avancées, http://www.imera.fr/.

46. Institut d'études avancées de Nantes, http://www.iea-nantes.fr/.

47. Institut d'études avancées de Paris, http://paris-iea.fr/.

48. Die Forscher des CNRS sind zum Großteil auf Lebenszeit verbeamtet. Deswegen und aus anderen Gründen ist der Vergleich mit der Deutschen Forschungsgemeinschaft nur begrenzt möglich.

49. Institut des sciences sociales et humaines du CNRS, http://www.cnrs.fr/inshs/.

50. Maisons des Sciences de l'Homme, http://www.msh-reseau.fr/.

51. Fondation Maison des sciences de l'homme, http://www.fmsh.fr/.

52. Vgl. alle Ergebnisse im Detail, »investissements d'avenir«, http://www.enseignementsuprecherche.gouv.fr/pid24578/investissements-d-avenir.html.

53. MATRICE, http://www.iscpif.fr/matrice-fr.

54. DIME-SHS, http://www.sciencespo.fr/dime-shs/.

55. BIBLISSIMA, http://www.biblissima-condorcet.fr/.

56. CAP, http://www.labex-hesam.eu/fr/7-cap-presentation.

57. PRES Hesam, HASTEC, http://www.labex-hesam.eu/fr/13-hastec-presentation.

58. PRES Hesam, LaScArBx, http://lascarbx.labex-univ-bordeaux.fr/.

59. RESMED, http://www.labex-resmed.fr/.

60. TRANSFERS, http://www.transfers.ens.fr/.

61. ARCHIMEDE, http://recherche.univ-montp3.fr/unites-de-recherche/22-labex-archeologie-ethistoire-de-la-mediterranee-et-de-legypte-anciennes-archimede/.

62. COMOD, http://pensee-classique.ens-lyon.fr/spip.php?article748.

63. EHNE, http://www.labex-ehne.fr/.

64. PP, http://passes-present.eu/fr.

65. TEPSIS, http://www.labex-hesam.eu/fr/presentation.

66. Online zugänglich auf: http://www.cairn.info/revue-hypotheses.htm. Vgl. auch die Webseite der Graduiertenschule der Universität Paris I: http://www.univ-paris1.fr/ecoles-doctorales/edhistoire/.

67. http://www.enseignementsup-recherche.gouv.fr/cid20185/le-doctorat.html.

68. Die komplette Aufstellung: https://appliweb.dgri.education.fr/annuaire/ListeSd.jsp? entite $=$ ed\&ds $=6 \&$ prov=ListeDs.

69. Die Geschichtswissenschaft ist oft nur ein Teilaspekt solcher Doktorandenschulen, die "Geschichte« im Titel führen und häufig auch die Literaturwissenschaft, die Kunstgeschichte, die Philosophie etc. betreffen. 
70. ANR, http://www.agence-nationale-recherche.fr/.

71. ANR, Deutsch-französisches Programm, http://www.agence-nationale-recherche.fr/financervotre-projet/appel-detail/appel-a-projets-franco-allemand-en-sciences-humaines-et-

sociales-2013/.

72. Agence d'Évaluation de la Recherche et de l'Enseignement Supérieur (AERES), http:// www.aeres-evaluation.fr/.

73. Loi LESR du MESR, http://www.enseignementsup-recherche.gouv.fr/pid29078/projet-de-loid-orientation-pour-l-e.s.r.html.

74. Deutsch-Französische Hochschule, http://www.dfh-ufa.org.

75. Vier auf Bachelor- bzw. licence-Niveau, vier auf Masterniveau und eine Kombination aus Master- und Doktorandenprogramm in der Form eines sogenannten PHD-Track.

76. IUF, http://iuf.amue.fr/.

77. École Normale Supérieure Paris, http://www.ens.fr/, École Normale Supérieure Lyon, http:// www.ens-lyon.eu/ecole-normale-superieure-de-lyon-accueil-77247.kjsp und École Normale Supérieure Cachan, http://www.ens-cachan.fr/.

78. École des Chartes, http://www.enc.sorbonne.fr/.

79. École Polytechnique, http://www.polytechnique.edu/.

80. ENPC, http://www.enpc.fr/.

81. Vgl. die Titel der Lehrveranstaltungen und Masterstudiengänge der EHESS, http:// www.ehess.fr/fr/enseignement/enseignements/2013/mention/ und der EPHE, http:// www.ephe.sorbonne.fr/categoryevents/1021.html.

82. CIERA, http://www.ciera.fr/ciera/.

83. INALCO, http://www.inalco.fr/.

84. Sciences Po, http://www.sciencespo.fr/.

85. Vgl. dazu auch den Beitrag von Jean-Louis Georget in diesem Wegweiser.

86. Sciences Po, http://www.sciencespo.fr/content/124/les-structures-juridiques.

87. Centre d'Études et de Recherches Internationales, http://www.sciencespo.fr/ceri/.

88. Centre d'Histoire de Sciences Po, http://chsp.sciences-po.fr/.

89. Centre de Recherches Politiques de Sciences Po, http://www.cevipof.com/.

90. Max Weber Stiftung, http://www.maxweberstiftung.de/.

91. École Française de Rome, http://www.ecole-francaise.it/fr/index.html.

92. École Française d'Athènes, http://www.efa.gr/accueil.htm.

93. Casa de Velasquez, http://www.casadevelazquez.org/.

94. Das hat zur Folge, dass 2014 ein Student in den classes préparatoires den Staat nach wie vor doppelt soviel kostet wie ein Bachelor-Student. Auch werden Lehrer in den classes préparatoires zu Beginn ihrer Karriere besser bezahlt als Hochschullehrer an den Universitäten oder Forscher im CNRS.

95. Historikerverband, http://www.historikerverband.de/verband/aufgaben-und-ziele.htm.

96. Geschichtslehrerverband, http://www.geschichtslehrerverband.org/.

97. Historikertag, http://www.historikertag.de.

98. Rendez-vous de l'histoire Blois, http://www.rdv-histoire.com/.

99. Rendez-vous de l'histoire Weimar, http://www.weimarer-rendezvous.de/.

100. Association des professeurs d'histoire et de géographie, http://www.aphg.fr/.

101. Siehe dazu auch den Beitrag von Claire Gantet in diesem Wegweiser (http:// ifha.revues.org/7857).

102. Société des Professeurs d'Histoire Ancienne à l'Université, http://sophau.univ-fcomte.fr/.

103. Société des Historiens Médiévistes de l'Enseignement Supérieur Public, http:// www.shmesp.fr/.

104. Association des Historiens Modernistes des Universités Françaises, http:// ahmuf.hypotheses.org/. 
105. Association des Historiens Contemporanéistes de l'Enseignement Supérieur et de la Recherche, http://ahcesr.hypotheses.org/.

106. Association des Germanistes de l'Enseignement Supérieur, http://www.ages-info.org/spip/.

107. Zum Programm der agrégation im Fach Deutsch gehörte beispielsweise 2013 das Thema: »Der Weg zur deutschen Einheit 1815-1871«.

108. Comité des Travaux Historiques et Scientifiques, http://cths.fr/.

109. 2014 wird sich die 139. Jahrestagung mit dem Thema "Sprache und Kommunikation« beschäftigen.

110. Collège de France, http://www.college-de-france.fr/site/college/index.htm.

111. Académie des Inscriptions et Belles Lettres, http://www.aibl.fr/.

\section{RÉSUMÉS}

Der Artikel präsentiert die französische Geschichtswissenschaft unter besonderer Berücksichtigung ihrer Institutionen, ihrer Finanzierung sowie ihrer gegenwärtigen Organisationsformen. Letztere sind durch vielfältige Strukturen und Einrichtungen geprägt, die für externe Beobachter oft nur schwer zu entschlüsseln sind. Der Beitrag versucht, diese Vielfalt, in der Universitäten, grandes écoles, Forschungseinrichtungen, grands établissements und zeitlich begrenzte Forschungsprogramme einander gegenüberstehen, historisch $\mathrm{zu}$ erklären. Anschließend werden die verschiedenen Reformen (vor allem der letzten zehn Jahre) betrachtet, deren Ziel es ist, die unterschiedlichen Strukturen und Akteure besser zu verbinden und einander anzunähern, wobei es bislang jedoch nicht gelungen ist, die Dichotomie zwischen universitären und außeruniversitären Einrichtungen zu überbrücken.

\section{INDEX}

Schlüsselwörter : Frankreich, Geschichtswissenschaft, Forschung, Universitäten, Hochschulen und Institute

\section{AUTEURS}

\section{PIERRE MONNET}

Prof. Dr. Pierre Monnet ist Directeur d'études an der École des Hautes Etudes en Sciences Sociales (EHESS) und leitet seit 2011 das Institut Français d'Histoire en Allemagne in Frankfurt a. M.

Mail: Monnet[at]ehess.fr 